\title{
BIO-POTENT SULFONAMIDES
}

\author{
SELVAKUMAR DINESHKUMAR AND GANESAMOORTHY THIRUNARAYANAN*
}

Department of Chemistry, Annamalai University, Annamalainagar-608 002, India

\begin{abstract}
Some 4-(substituted phenylsulfonamido)benzoic acids have been synthesized by fly-ash: $\mathrm{H}_{3} \mathrm{PO}_{3}$ nano catalyst catalyzed condensation of substituted benzenesulfonyl chlorides and 4-aminobenzoic acid in ultrasound irradiation conditions. The yields of the sulfonamides are more than $90 \%$. The synthesized 4-(substituted phenylsulfonamido) benzoic acid derivatives were characterized by their physical constants, analytical and spectroscopic data. Antimicrobial activities of all sulfonamides were measured by Bauer-Kirby disc diffusion method.
\end{abstract}

Key words: 4-(substitutedphenylsulfonamido)benzoic acids; Ultrasonication; Antimicrobial activities.

\section{INTRODUCTION}

Sulfonamides or sulphonamide are effective compounds combining $\left(-\mathrm{S}(=\mathrm{O})_{2}-\mathrm{NH}-\right)$ group and they are generally formed by the condensation of corresponding amine and sulfonyl chlorides. The most common method for synthesizing sulfonamides is the condensation of aromatic sulfonyl chloride with aliphatic or aromatic primary/secondary amines in the presence of base catalysts. Usually sulfonamides are derived from amines with sulfonic acids or sulfonyl halides by eradicating of halide group or hydroxyl group and form important class of sulfur holding sulfonamides. Researchers have been investigated various compounds containing sulfonamide moieties. They are tryptamine $^{1}$, chalcones ${ }^{2}$, adamantine ${ }^{3}$, azetidinone ${ }^{4}$, carbazole $^{5}$, catenane ${ }^{6}$, cinnamic acyl ${ }^{7}$, coumarin ${ }^{8}$, cyanopyridines, isoxazoles, pyrazoles, pyrimidines ${ }^{9}$, epipodophyllotoxin ${ }^{10}$, furan containing vinyl sulfonamides ${ }^{11}$, isoquinoline sulfonamide ${ }^{12}$, oxazolidinones ${ }^{13}$, quinolone ${ }^{14}$, thiadiazole ${ }^{15}$, thiazole,pyridone, chromene, hydrazine ${ }^{16}$, thiouracil ${ }^{17}$ and triazole ${ }^{18}$. Due to its greater effectiveness and less toxicity it establishes a main class of drugs with numerous types of pharmacological potentials such as antibacterial ${ }^{19}$, antitumor ${ }^{20}$, anti-carbonic anhydrase $^{21-23}$, antiretro-viral ${ }^{24}$, anti-malarial ${ }^{25}$, anti-HIV[26]. Many catalysts are used for the synthesis of sulfonamides such as, $\beta$-Cyclodextrin ${ }^{27}$, water ${ }^{28}$, ${ }^{29}$, pyridine ${ }^{30}, \mathrm{PPh}_{3}{ }^{31}, \mathrm{DMAP}^{32}, \mathrm{CsF}-\mathrm{Celite}^{33}, \mathrm{ZnO}$-nanoparticles ${ }^{34}, \mathrm{MgO}^{35}$. Lakroutet al., ${ }^{36}$ have prepared somesulfonamide derivatives by sulfonylation of primary, secondary sulfonyl chlorides, amino acid esters and phenols under solvent-free microwave irradiation techniques. Pan et al., ${ }^{28}$ synthesized a fewsulfonamides via $I_{2}$-mediated reaction of sodium sulfinates with amines in an aqueous medium at room temperature. Recently Dineshkumar and Thirunarayanan prepared more than $80 \%$ yields of mesalazines type sulphonamides ${ }^{37}$. In the literature surey, there is no report available for the ultrasonic assisted fly-ash: $\mathrm{H}_{3} \mathrm{PO}_{3}$ nano catalyst ${ }^{38}$ catalyzed condensation of substituted benzenesulfonyl chlorides and 4-aminobenzoic acid in ultrasonication under solvent-free conditions and its biological activities Therefore herein the authors interestingly investigated the ultrasound assisted synthesis of some sulphonamides and evaluation of antimicrobial activities using the standard Bauer-Kirby ${ }^{39}$ Disc diffusion method.

\section{EXPERIMENTAL}

\section{General}

Sigma-Aldrich, Alfa Aesar and E-Merck chemical company chemicals used in this investigation. Mettler FP51 melting point apparatus was used for the determination melting points of all sulfonamides. The OMNIC Fouriertransform spectrophotometer was employed for recording Infrared spectra. The proton and carbon-13 spectra of all sulfonamides were recorded in Bruker AV400 \& 500 NMR spectrometer in DMSO solvent using TMS as internal standard. Mass spectra were recorded on a SIMADZU GC-MS2010 spectrometer. The Thermo Finnigan $\mathrm{CHN}$ analyzer was used for elemental analysis.

Typical procedure for the preparation of 4-(substituted phenylsulfonamido) benzoic acids

An equimolar concentration of benzenesulfonyl chloride $(1 \mathrm{mmol})$ 4-aminobenzoic acid (1 mmol), fly-ash: $\mathrm{H}_{3} \mathrm{PO}_{4}(0.02 \mathrm{mg})$ catalyst $^{38}$ and 10 $\mathrm{mL}$ of ethanol were taken in $50 \mathrm{~mL}$ conical flask and mixed thoroughly. This mixture was subjected to ultrasound irradiation for 20-25 minutes in a ultrasonicator (Scheme 1) in room temperature. During the reaction $0.1 \mathrm{mg}$ of potassium carbonate was added to neutralize the formation of hydrochloride. The completion of the reaction was monitored by Thin layer chromatography. The resulting product was washed with $n$-hexane and separated the catalyst by filtration and dried to obtain the solids. Further the crude was purified by column chromatogram using dichloromethane and ethylacetate $(3: 1)$ as eluants.<smiles>[X]c1ccc(S(=O)(=O)Cl)cc1</smiles>

$\underset{\text { Fly-ash: } \mathrm{H}_{3} \mathrm{PO}_{4}}{\longrightarrow}$
$\underset{\mathrm{C}_{2} \mathrm{H}_{5} \mathrm{OH},}{\text { Ultrasound }}$

Scheme-1 . Synthesis of 4-(substitutedphenylsulfonamido)benzoic acids<smiles>[X]c1cccc(S(=O)(=O)N(c2ccc(C(=O)O)cc2)c2ccc([N+](=O)[O-])cc2)c1</smiles> 
Measurement of antimicrobial activities

Measurement of antibacterial activities

Antibacterial activities of all synthesized sulphonamides were evaluated by using standard wellknown Bauer-Kirby disc diffusion method ${ }^{37}$. In this study the authors chosen the Gram-positive bacterial strains such as Micrococcus luteus B. subtilis and Staphylococcus aureus the Gram-negative bacterial strains such as E. coli, P.aeruginosa. Ampicillin as standard and the solvent is dimethylsulfoxide.

Measurement of antifungal activities

Antifungal activities of all synthesized mesalazine sulfonamides were determined by using standard wellknown Bauer-Kirby disc diffusion method ${ }^{37}$. The test organisms were subculture using PDA medium. In this study, the authors had chosen two fungal stains such as Aspergillus niger and Trichoderma viride. Micanazole was used as a standard drug and dimethylsulfoxide as a solvent.

\section{RESULTS AND DISCUSSION}

In our research laboratory, we attempt to synthesis some sulphonamides using fly-ash: $\mathrm{H}_{3} \mathrm{PO}_{4}$ catalyst assisted ultrasonication method. An appropriate molar quantities of electron donating and electron-withdrawing substituted benzenesulfonyl chloride (1 mmol), 4-aminobenzoic acid (1 mmol), flyash: $\mathrm{H}_{3} \mathrm{PO}_{4}(0.02 \mathrm{mg})$ catalyst and $10 \mathrm{~mL}$ of ethanol were taken in $50 \mathrm{~mL}$ conical flask and mixed thoroughly. This mixture was subjected to ultrasound irradiation for 20-25 minutes in a ultrasonicator in room temperature. During the reaction $0.1 \mathrm{mg}$ of potassium carbonate was added to neutralize the formation of hydrochloride. The completion of the reaction was monitored by Thin Layer Chromatography. The resulting product was washed with $n$-hexane and separated the catalyst by filtration and dried to obtained $90-96 \%$ yields of sulphonamide derivatives. The electron donating substituted benzene sulfonyl chlorides gave higher percent yields than the compound containing electronwithdrawing substituents. The complete characterization data of synthesized sulphonamide derivatives (entries 1-9) are summarized below

4-(Phenylsulfonamido)benzoic acid (1): White solid, m.p. $276^{\circ} \mathrm{C}$. IR(4000-400, v, $\left.\mathrm{cm}^{-1}\right)$ : 3272(NH), 1677(CO), 1332(SOsym), 1163(SO asym). ${ }^{1} \mathrm{H}$ NMR ( ppm): 10.824(s, 1H, NH), 12.750(s, 1H, COOH), 7.200-7.843(m, $9 \mathrm{H}, \mathrm{Ar}-\mathrm{H}) ;{ }^{13} \mathrm{C}$ NMR ( ppm): 167.19(CO), 118.63-142.40(Ar-C). Anal. Calcd. forC $\mathrm{H}_{13} \mathrm{H}_{11} \mathrm{NO}_{4} \mathrm{~S}(\%): \mathrm{C}(56.31), \mathrm{H}(4.00), \mathrm{N}(5.05)$; Found (\%):C (56.38), $\mathrm{H}$ (4.05), N (4.98); MW: 277; Mass (m/z): $277\left[\mathrm{M}^{+}\right]$

4-((4-Bromophenyl)sulfonamido)benzoic acid(2): White solid, m.p. 292-293 ${ }^{\circ} \mathrm{C}$. IR(4000-400, v, $\left.\mathrm{cm}^{-1}\right): 3263(\mathrm{NH}), 1680(\mathrm{CO}), 1337$ (SOsymm), 1161(SOasym). ${ }^{1} \mathrm{H}$ NMR ( ppm): 10.913(s, 1H, NH), 11.326(s, 1H, COOH), 7.234-7.7364(m, 8H, Ar-H). ${ }^{13} \mathrm{C}$ NMR ( ppm): 167.19(CO), 119.23-144.23(ArC). Anal. Calcd.forC ${ }_{13} \mathrm{H}_{10} \mathrm{BrNO}_{4} \mathrm{~S}(\%)$ : C (43.84), H (2.83), N (3.93); Found (\%):C (43.86), H (2.87), N (3.89); MW: 356; Mass (m/z): $356\left[\mathrm{M}^{+}\right], 358\left[\mathrm{M}^{2+}\right]$. 4-((4-chlorophenyl)sulfonamido)benzoic acid(3): White solid, m.p. 285-287 ${ }^{\circ} \mathrm{C}$. IR $\left(4000-400, v, \mathrm{~cm}^{-1}\right): 3258(\mathrm{NH}), 1678(\mathrm{CO}), 1336(\mathrm{SO}$ sym), 1161(SOasym). ${ }^{1} \mathrm{H}$ NMR ( ppm): 10.624(s, 1H, NH), 11.901(s, 1H, COOH), 7.134-7.726(m, 8H, Ar-H). ${ }^{13} \mathrm{C}$ NMR ( ppm): 167.16(CO), 118.502-144.93(Ar-
C). Anal. Calcd.forC $\mathrm{H}_{10}$ BrNO S (\%): $\mathrm{C}$ (50.09), $\mathrm{H}$ (3.23), N (4.49); Found (\%):C (50.12), H (3.20), N (4.47); MW: 311; Mass (m/z): $311\left[\mathrm{M}^{+}\right], 313\left[\mathrm{M}^{2+}\right]$. 4-((2-fluorophenyl)sulfonamido)benzoic acid(4): White powder, m.p. 267-268 ${ }^{\circ} \mathrm{C} . \quad \operatorname{IR}\left(4000-400, v, \mathrm{~cm}^{-1}\right): 3258(\mathrm{NH}), 1700(\mathrm{CO}), 1336(\mathrm{SOsym})$, 1150(SOasym). ${ }^{1} \mathrm{H}$ NMR ( ppm): 10.364(s, 1H, NH), 12.004(s, 1H, COOH), 7.301-7.749(m, 8H, Ar-H). ${ }^{13} \mathrm{C}$ NMR ( ppm):167.50(CO), 118.365-143.78(ArC). Anal. Calcd.forC $\mathrm{H}$ FNO S (\%): C (52.88), H (3.41), N (4.74); Found (\%):C (52.92), H (3.38), N (4.71); MW: 295; Mass (m/z): $295\left[\mathrm{M}^{+}\right], 297\left[\mathrm{M}^{2+}\right]$. 4-((4-fluorophenyl)sulfonamido)benzoic acid(5): White solid, m.p. 270-272 ${ }^{\circ} \mathrm{C}$. IR(4000-400, v, $\left.\mathrm{cm}^{-1}\right): 3247(\mathrm{NH}), 1693(\mathrm{CO}), 1334(\mathrm{SOsym})$, 1159(SOasym). ${ }^{1} \mathrm{H}$ NMR ( ppm): 10.083(s, $\left.1 \mathrm{H}, \mathrm{NH}\right), 11.043(\mathrm{~s}, 1 \mathrm{H}, \mathrm{COOH})$, 7.435-7.782(m, 8H, Ar-H). ${ }^{13} \mathrm{C}$ NMR ( ppm): 167.21(CO), 118.093-142.87(ArC). Anal. Calcd.forC $\mathrm{H}_{10} \mathrm{FNO} \mathrm{S}(\%)$ : C (52.88), H (3.41), N (4.74); Found (\%):C (52.96), H (3.36), N (4.72); MW: 295; Mass (m/z): $295\left[\mathrm{M}^{+}\right], 297\left[\mathrm{M}^{2+}\right]$. 4-((4-methoxyphenyl)sulfonamido)benzoic acid(6): White solid, m.p. $278-281^{\circ} \mathrm{C}$. IR(4000-400, v, $\left.\mathrm{cm}^{-1}\right)$ : 3264(NH), 1700(CO), 1332(SOsym), 1150(SOasym). ${ }^{1} \mathrm{H}$ NMR ( ppm): 10.700(s, $\left.1 \mathrm{H}, \mathrm{NH}\right), 10.002(\mathrm{~s}, 1 \mathrm{H}, \mathrm{COOH})$, 2.504(s, 3H, CH ) , 7.216-7.693(m, 8H, Ar-H). ${ }^{13} \mathrm{C} \mathrm{NMR} \mathrm{(ppm):} \mathrm{167.26(CO),}$ 63.87( $\left(\mathrm{OCH}_{3}\right)$, 118.02-144.06(Ar-C). Anal. Calcd.forC ${ }_{14} \mathrm{H}_{13} \mathrm{NO}_{5} \mathrm{~S}(\%): \mathrm{C}$ (54.72), H (3.41), N (4.74); Found (\%):C (54.76), H (4.22), N (4.54); MW: 307; Mass (m/z): $307\left[\mathrm{M}^{+}\right]$

4-((4-methylphenyl)sulfonamido)benzoic acid(7): White powder, m.p. 265-267 ${ }^{\circ}$. IR $\left(4000-400, v, \mathrm{~cm}^{-1}\right): 3215(\mathrm{NH}), \quad 1704(\mathrm{CO}), \quad 1336(\mathrm{SOsym})$ 1154(SOasym). ${ }^{1} \mathrm{H}$ NMR ( ppm): 11.116(s, 1H, NH), 12.348(s,1H, COOH), 2.083(s,3H, $\left.\mathrm{CH}_{3}\right), 7.381-7.762(\mathrm{~m}, 8 \mathrm{H}, \mathrm{Ar}-\mathrm{H}) .{ }^{13} \mathrm{C}$ NMR ( ppm): 167.17(CO), 22.46( $\left.\mathrm{CH}_{3}\right), 118.12-143-36\left(\right.$ Ar-C). Anal. Calcd.forC ${ }_{14} \mathrm{H}_{13} \mathrm{NO}_{4} \mathrm{~S}(\%)$ : C (57.72), H (4.50), N (4.81); Found (\%):C (57.79), H (4.48), N (4.78); MW: 291; Mass $(\mathrm{m} / \mathrm{z}): 291\left[\mathrm{M}^{+}\right]$.

4-((2-nitrophenyl)sulfonamido)benzoic acid(8): White solid, m.p.280$282^{\circ} \mathrm{C}$. IR(4000-400, v, $\left.\mathrm{cm}^{-1}\right): 3213(\mathrm{NH}), \quad 1697(\mathrm{CO}), \quad 1336(\mathrm{SOsym})$, 1162(SOasym). ${ }^{1} \mathrm{H}$ NMR ( ppm): 11.234(s, 1H, NH), 11.471(s, 1H, COOH), 7.435-7.892(m, 8H, Ar-H). ${ }^{13} \mathrm{C} \mathrm{NMR}$ ( ppm): 167.14(CO), 118.56-144.25(ArC). Anal. Calcd.forC ${ }_{13} \mathrm{H}_{10} \mathrm{~N}_{2} \mathrm{O} S(\%)$ : C (48.45), $\mathrm{H}$ (3.13), N (8.69); Found (\%):C (48.49), H (3.09), N (8.62); MW: 322; Mass (m/z): $322\left[\mathrm{M}^{+}\right]$.

4-((4-nitrophenyl)sulfonamido)benzoic acid(9): White solid, m.p.275$276^{\circ} \mathrm{C}$. IR(4000-400, v, $\left.\mathrm{cm}^{-1}\right): 3257(\mathrm{NH}), \quad 1677(\mathrm{CO}), \quad 1338(\mathrm{SOsym})$, 1162(SOasym). ${ }^{1} \mathrm{H}$ NMR ( ppm): 11.128(s, 1H, NH), 11.5.308(s, 1H, COOH), 7.465-7.832(m, 8H, Ar-H). ${ }^{13} \mathrm{C}$ NMR ( ppm): 167.13(CO), 119.09-144.83(ArC). Anal. Calcd.forC $\mathrm{H}_{13} \mathrm{~N}_{2} \mathrm{O}_{6} \mathrm{~S}(\%): \mathrm{C}(48.45), \mathrm{H}(3.13), \mathrm{N}(8.69)$; Found (\%):C (48.46), H (3.10), N (8.64); MW: 322; Mass (m/z): $322\left[\mathrm{M}^{+}\right]$.

Authors have studied this experiment with methods of conventional heating for $6 \mathrm{~h}$ and microwave irradiation for 3-5 minutes for the condensation of benzene sulfonyl chlorides and 4-aminobenzoic acid(entries 1-9). In these methods the obtained yields are less than $80 \%$. The obtained yields (entries 1-9) in conventional heating, microwave irradiation and ultrasonication methods are presented in Table 1. Among the three methods, the ultrasonication synthesis gave high yields than other two methods.

Table-1. The obtained yields of sulphonamides in the various method of synthesis.

\begin{tabular}{|c|c|c|c|c|c|c|c|}
\hline \multirow{2}{*}{ Entry } & \multirow{2}{*}{$\mathrm{X}$} & \multicolumn{2}{|c|}{ Conventional heating } & \multicolumn{2}{|c|}{ Microwave irradiation } & \multicolumn{2}{|c|}{ Ultrasound irradiation } \\
\hline & & Time(h) & Yield (\%) & Time(m) & Yield (\%) & Time(m) & Yield (\%) \\
\hline 1 & $\mathrm{H}$ & 6 & 68 & 3 & 75 & 20 & 94 \\
\hline 2 & 4-Br & 6 & 65 & 4 & 70 & 25 & 92 \\
\hline 3 & $4-\mathrm{Cl}$ & 6 & 66 & 4 & 71 & 25 & 91 \\
\hline 4 & $2-\mathrm{F}$ & 6 & 63 & 5 & 73 & 23 & 90 \\
\hline 5 & $4-\mathrm{F}$ & 6 & 67 & 3.5 & 74 & 21 & 90 \\
\hline 6 & $4-\mathrm{OCH}_{3}$ & 6 & 72 & 3 & 78 & 25 & 96 \\
\hline 7 & $4-\mathrm{CH}_{3}$ & 6 & 70 & 3 & 76 & 25 & 94 \\
\hline 8 & $2-\mathrm{NO}_{2}$ & 6 & 61 & 5 & 68 & 24 & 90 \\
\hline 9 & $4-\mathrm{NO}_{2}$ & 6 & 61 & 5 & 71 & 22 & 91 \\
\hline
\end{tabular}

Also the authors studied the effects of solvent on these synthesis of sulphonamides in the above three methods within the same conditions such as quantity of catalyst and reaction time. The solvents such as methanol, ethanol, dichloromethane, tetrahydrofuron and dioxane were used for the evaluation of effects of solvent on synthesis of sulphonamides. Here the electron donating substituted sulfonylchlorides gave more percentage yield than electron with- drawing substituted sulfonylchlorides. In overall, among the three methods the ultrasonication synthetic method gave more yields than conventional heating method. The obtained yields of the sulphonamuides with various solvents in the conventional heating, and ultrasonic sound irradiation methods are presented in Table 2 . 
Table-2. The effects of solvents on the obtained yields of sulphonamides in conventional and ultrasound irradiation method of synthesis.

\begin{tabular}{|c|c|c|c|c|c|c|c|c|c|c|c|c|c|}
\hline \multirow{2}{*}{ Entry } & \multirow{2}{*}{$\mathrm{X}$} & \multicolumn{6}{|c|}{ Yields $(\%)$ in Conventional heating with solvents } & \multicolumn{6}{|c|}{ Yields (\%) in Ultrasound irradiation with solvents } \\
\hline & & $\mathrm{MeOH}$ & $\mathrm{EtOH}$ & DCM & THF & DX & $\mathrm{ACN}$ & $\mathrm{MeOH}$ & EtOH & DCM & THF & DX & $\mathrm{ACN}$ \\
\hline 1 & $\mathrm{H}$ & 67 & 68 & 66 & 68 & 69 & 69 & 90 & 94 & 91 & 90 & 91 & 90 \\
\hline 2 & $4-\mathrm{Br}$ & 66 & 65 & 65 & 64 & 62 & 65 & 92 & 92 & 93 & 95 & 93 & 91 \\
\hline 3 & $4-\mathrm{Cl}$ & 63 & 66 & 64 & 66 & 65 & 70 & 95 & 91 & 94 & 94 & 93 & 95 \\
\hline 4 & $2-F$ & 62 & 63 & 63 & 62 & 61 & 71 & 91 & 90 & 91 & 93 & 91 & 92 \\
\hline 5 & $4-\mathrm{F}$ & 63 & 67 & 65 & 64 & 63 & 68 & 93 & 90 & 93 & 92 & 92 & 92 \\
\hline 6 & $4-\mathrm{OCH}_{3}$ & 70 & 72 & 68 & 70 & 72 & 74 & 96 & 96 & 95 & 96 & 95 & 96 \\
\hline 7 & $4-\mathrm{CH}_{3}$ & 66 & 70 & 67 & 69 & 68 & 69 & 93 & 94 & 94 & 93 & 94 & 93 \\
\hline 8 & $2-\mathrm{NO}_{2}$ & 63 & 61 & 62 & 63 & 62 & 66 & 91 & 90 & 90 & 92 & 91 & 90 \\
\hline 9 & $4-\mathrm{NO}_{2}$ & 62 & 61 & 63 & 65 & 65 & 65 & 91 & 91 & 92 & 93 & 94 & 92 \\
\hline
\end{tabular}

MeOH: Methanol; EtOH: Ethanol; DCM: Dichloromethane; THF: Tetrahydrofuron; DX: Dioxane; ACN: Acetonitrile

\section{Antibacterial activity}

The measured antibacterial activities by means of the measurement of $\mathrm{mm}$ of zone of inhibitions $\mathrm{s}^{39-41}$ of the synthesized sulfonmamides are presented in Table 3. The mm of zone of inhibition in the petri-plates (plates 1-8) are shown in Fig. 2. The comparative statistical diagram of $\mathrm{mm}$ of zone of inhibition was illustrated in Fig. 3.

Table-3.Antibacterial activities of 4-(substituted phenylsulfonamido) benzoic acids.

\begin{tabular}{|c|c|c|c|c|c|}
\hline \multirow{3}{*}{ Entry } & \multirow{3}{*}{$\mathrm{X}$} & \multicolumn{4}{|c|}{ Zone of inhibition (mm) } \\
\hline & & \multicolumn{2}{|c|}{ Gram-positive } & \multicolumn{2}{|c|}{ Gram-negative } \\
\hline & & M. luteus & S. aureus & E. coli & $\begin{array}{c}P . \\
\text { aeruginosa }\end{array}$ \\
\hline 1 & $\mathrm{H}$ & - & 6 & 6 & - \\
\hline 2 & $4-\mathrm{Br}$ & 6 & 6 & 7 & - \\
\hline 3 & $4-\mathrm{Cl}$ & 7 & 7 & 8 & 6 \\
\hline 4 & $2-\mathrm{F}$ & 6 & 7 & 9 & 8 \\
\hline 5 & $4-\mathrm{F}$ & 6 & - & 7 & - \\
\hline 6 & $4-\mathrm{OCH}_{3}$ & 9 & 7 & 7 & 11 \\
\hline 7 & $4-\mathrm{CH}_{3}$ & 7 & 7 & 8 & 7 \\
\hline 8 & $2-\mathrm{NO}_{2}$ & 7 & 7 & 12 & 8 \\
\hline 9 & $4-\mathrm{NO}_{2}$ & 8 & 10 & 9 & 16 \\
\hline Standard & Ampicillin & 17 & 24 & 20 & 19 \\
\hline Control & DMSO & - & - & - & - \\
\hline
\end{tabular}

The synthesized 4-(substituted phenylsulfonamido)benzoic acid compounds with 4- $\mathrm{OCH}_{3}$ substituent shows satisfactory antibacterial activity, the substituents 4-Br, 4-Cl, 2-F, 4-F, 4- $\mathrm{CH}_{3}, 2-\mathrm{NO}_{2}$ and 4- $\mathrm{NO}_{2}$ have shown least activity and the other substituted compounds have no antibacterial activity against Micrococcus luteus.

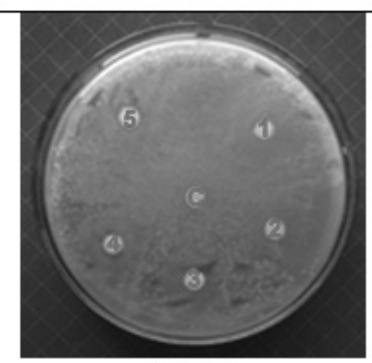

Plate 1

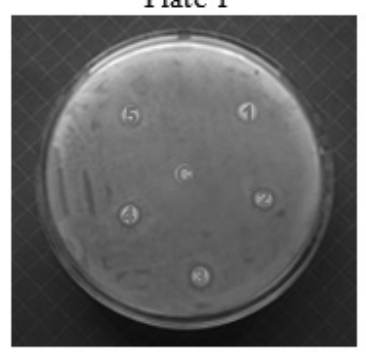

Plate 3

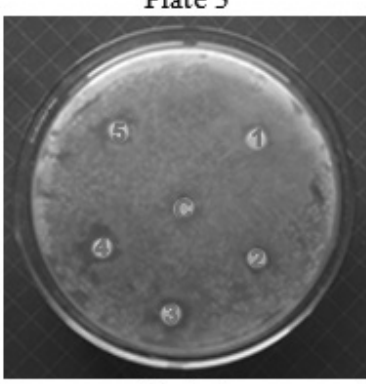

Plate 5

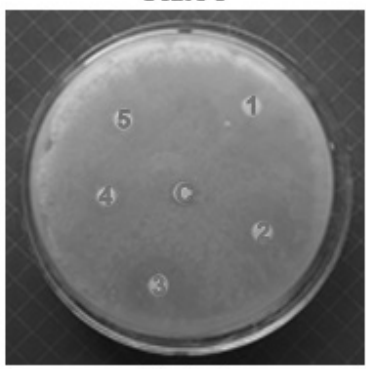

Plate 7

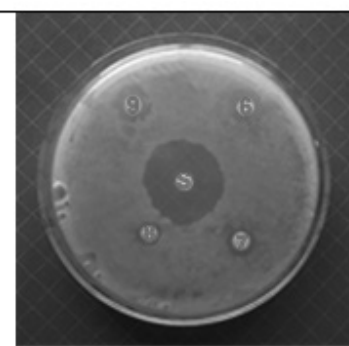

Plate 2

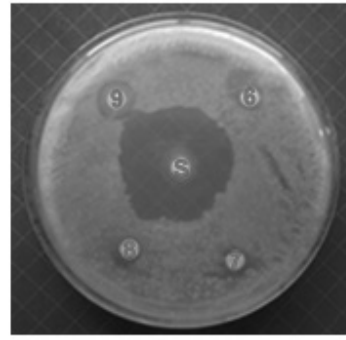

Plate 4

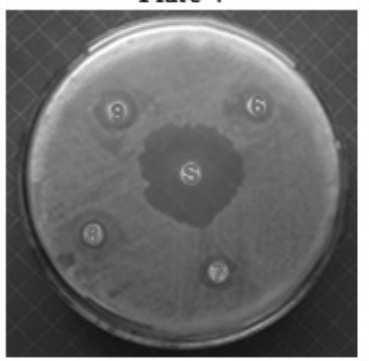

Plate 6

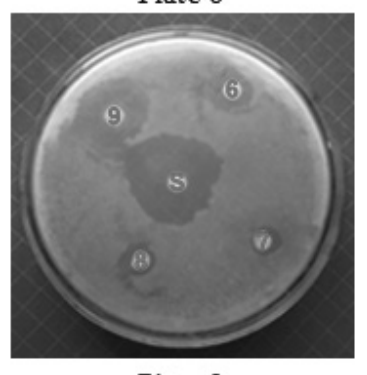

Plate 8

Fig 2. Antibacterial activities (petri-dishes) of 4-(substitutedphenylsulfonamido)benzoic acid compounds. 
The 4-(substituted phenylsulfonamido)benzoic acid compounds (with parent, 4-Br, 4-Cl, 2-F, 4-F, 4-- $\mathrm{OCH}_{3}, 4-\mathrm{CH}_{3}, 2-\mathrm{NO}_{2}$ and 4-NO have shown least antibacterial activity against Streptococcus aureus.

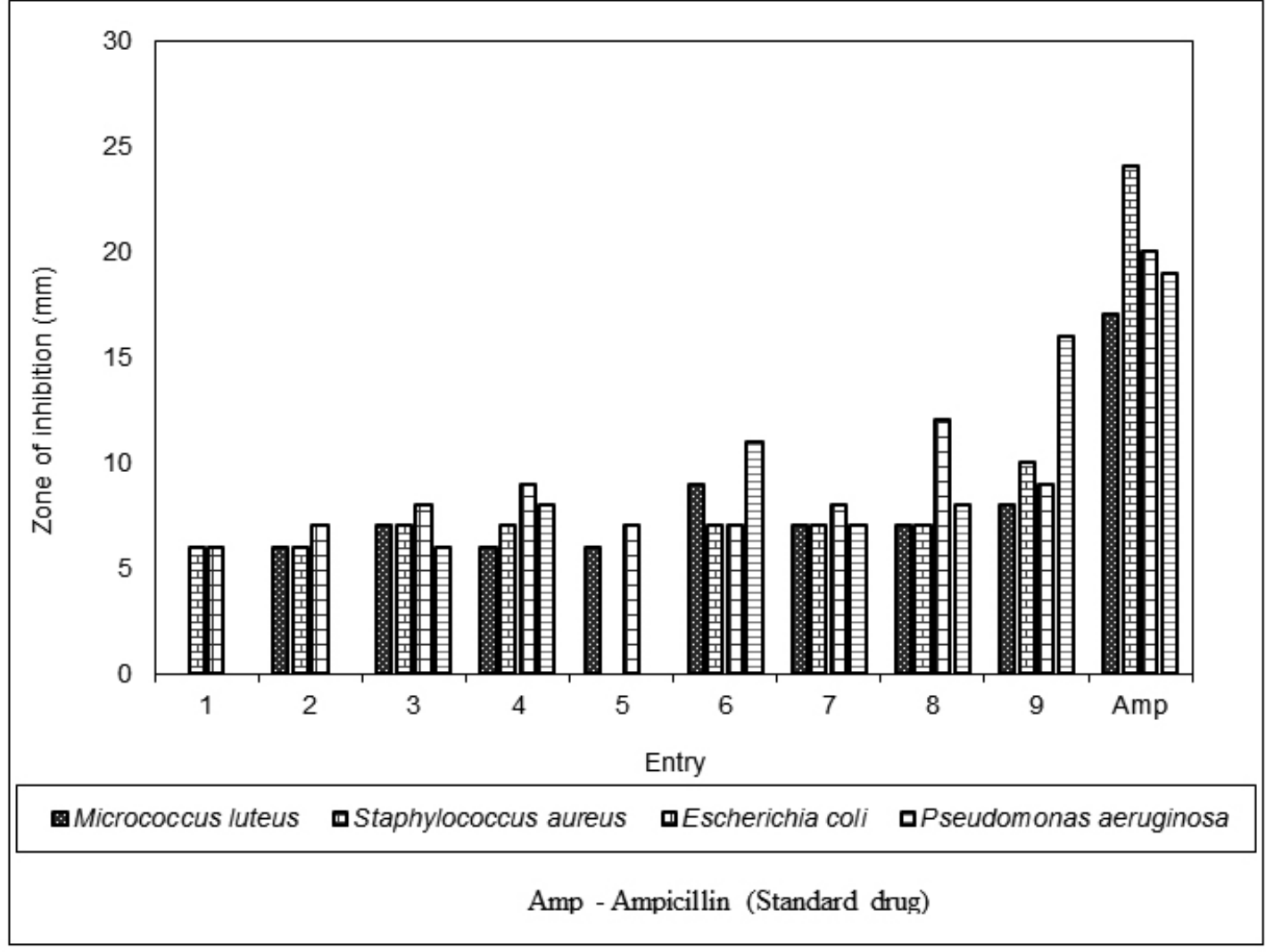

Fig. 3. Antibacterial activities (clustered column chart) of 4-(substituted phenylsulfonamido)benzoic acid compounds .

\section{Antifungal activity}

The antifungal activity of 4-(substituted phenylsulfonamido)benzoic acid compounds were shown(plates 9-12) in Fig 4. and the zone of inhibition ${ }^{39-41}$ values are given in $\mathbf{4}$. The corresponding clustered column chart is shown in Fig.5. The 4-(substituted phenylsulfonamido)benzoic acid compounds with 4- $\mathrm{Br}$ and 4- $\mathrm{CH}_{3}$ substituents have shown good antifungal activity and the compounds with parent, $2-\mathrm{F}$ and $4-\mathrm{F}$ substituent have shown satisfactory antifungal activity and the other substituted compounds no antifungal activity against Aspergillus niger.

Table-4. Antifungal activities of 4-(substituted phenylsulfonamido) benzoic acid compounds

\begin{tabular}{|c|c|c|c|}
\hline \multirow{2}{*}{ Entry } & \multirow{2}{*}{$\mathrm{X}$} & \multicolumn{2}{|c|}{ Zone of inhibition (mm) } \\
\cline { 3 - 4 } & $\mathrm{H}$ & $\begin{array}{c}\text { Aspergillus } \\
\text { niger }\end{array}$ & Trichodermaviride \\
\hline 1 & $4-\mathrm{Br}$ & 8 & 9 \\
\hline 2 & $4-\mathrm{Cl}$ & - & 13 \\
\hline 3 & $2-\mathrm{F}$ & 6 & 10 \\
\hline 4 & $4-\mathrm{F}$ & 7 & 14 \\
\hline 5 & $4-\mathrm{OCH}$ & - & 9 \\
\hline 6 & $4-\mathrm{CH}_{3}$ & 8 & 13 \\
\hline 7 & $2-\mathrm{NO}_{2}$ & - & 12 \\
\hline 8 & $4-\mathrm{NO}_{2}$ & - & 8 \\
\hline 9 & Miconazole & 12 & 20 \\
\hline Standard & $\mathrm{DMSO}^{2}$ & - & - \\
\hline Control & & & \\
\hline
\end{tabular}

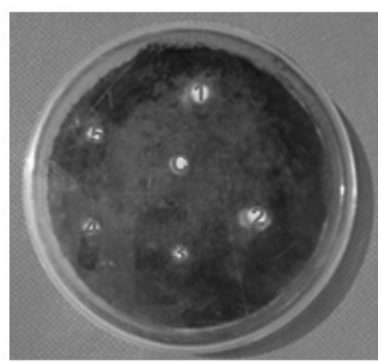

Plate 9

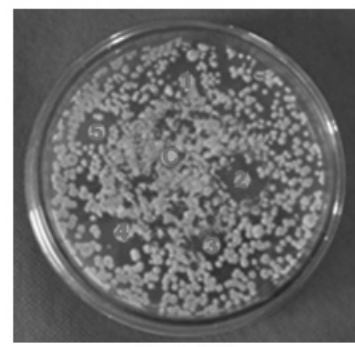

Plate 11

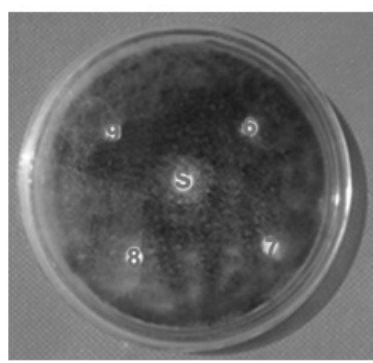

Plate 10

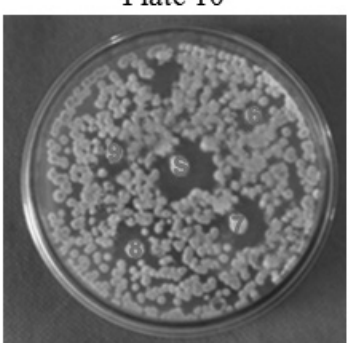

Plate 12
Fig 4. Antifungal activities of 4-(substituted phenylsulfonamido)benzoic acid compounds 


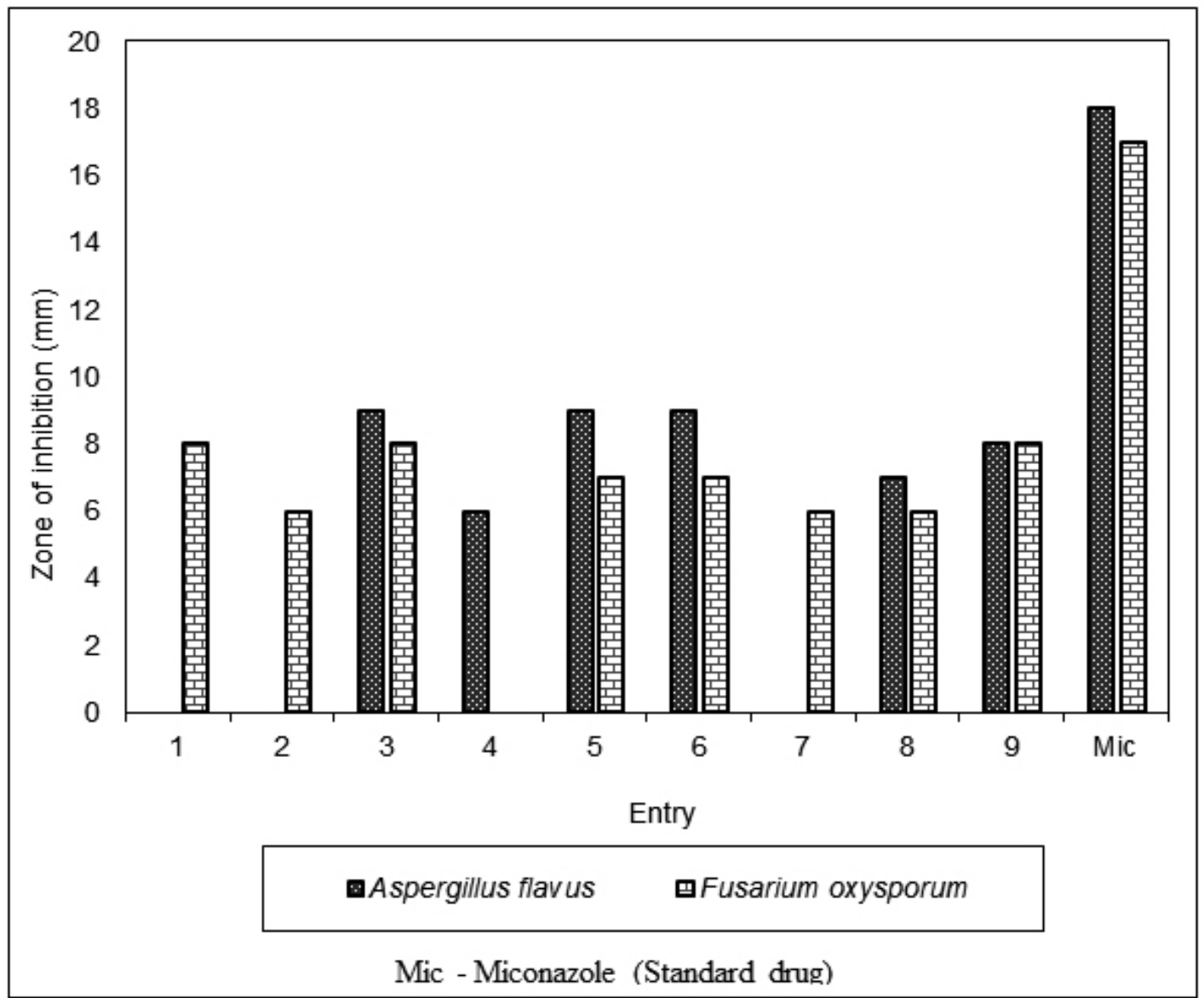

Fig.5. Antifungal activities (clustered column chart) of 4-(substituted phenylsulfonamido)benzoic acid compounds

The 4-(substituted phenylsulfonamido)benzoic acid compounds with 4-Br, 2-F, 4- $\mathrm{CH}_{3}$ and 2- $\mathrm{NO}_{2}$ substituents have shown good antifungal activity and the compounds with 4-Cl substituent have shown satisfactory antifungal activity and the other substituted compounds have shown least antifungal activity against Trichoderma viride.

\section{CONCLUSIONS}

The authors have demonstrated the ultrasonicated synthesis of some 4-(substituted phenylsulfonamido)benzoic acids by fly-ash: $\mathrm{H}_{3} \mathrm{PO}_{3}$ nano catalyst catalyzed condensation of substituted benzenesulfonyl chlorides and 4-aminobenzoic acid. The yields of the sulfonamides are more than $90 \%$. The synthesized 4-(substituted phenylsulfonamido) benzoic acid derivatives were characterized by their physical constants, analytical and spectroscopic data. Antimicrobial activities of all sulfonamides were measured by Bauer-Kirby disc diffusion method. compounds with $4-\mathrm{OCH}_{3}$ substituent shows satisfactory antibacterial activity, the substituents 4- $\mathrm{Br}, 4-\mathrm{Cl}, 2-\mathrm{F}, 4-\mathrm{F}, 4-\mathrm{CH}_{3}, 2-\mathrm{NO}_{2}$ and 4-NO have shown least activity and the other substituted compounds have no antibacterial activity against Micrococcus luteus. The 4-(substituted phenylsulfonamido)benzoic acids with parent, 4-Br, 4-Cl, 2-F, 4-F, 4- $\mathrm{OCH}_{3}$, $4-\mathrm{CH}_{3}, 2-\mathrm{NO}_{2}$ and $4-\mathrm{NO}_{2}$ have shown least antibacterial activity against Streptococcus aureus. Compounds with $4-\mathrm{Br}$ and $4-\mathrm{CH}_{3}$ substituents have shown good antifungal activity and the compounds with parent, 2-F and 4-F substituent have shown satisfactory antifungal activity and the other substituted compounds no antifungal activity against Aspergillus niger. The 4-(substituted phenylsulfonamido)benzoic acids with 4-Br, 2-F, 4- $\mathrm{CH}_{3}$ and $2-\mathrm{NO}_{2}$ substituents have shown good antifungal activity and the compounds with $4-\mathrm{Cl}$ substituent have shown satisfactory antifungal activity. The other sulphonamides have shown least antifungal activity against Trichoderma viride.

\section{ACKNOWLEDGEMENT}

Authors thank DST-NMR Facility, Department of Chemistry, Annamalai University, Annamalainagar-608002 for recording NMR spectra of compounds.

\section{REFERENCES}

[1] T. Narasaiah, D. S. Rao, K. V. Ramana, S. Adam, C. N. Raju, Der Pharma Chemica. 4 1582, (2012).

[2] C. R. Andrighetti-Frohner, K. N. de Oliveira, D. Gaspar-Silva, L. K. Pacheco, A. C. Joussef, M. Steindel, C. M. O. Simoes, A. M. T. Souza, U. O. Magalhaes, I. F. Afonso, C. R.Rodrigues, R. J. Nunes, H. C. Castro, Eur. J. Med. Chem. 44, 755 (2009).

[3] G. L. Perlovich, T. V. Volkova, A. V. Sharapova, V. P. Kazachenko, N. N. Strakhova, A. N. Proshin, Phys. Chem. Chem. Phys. 18, 9281 (2016).

[4] X. Yuan, P. Lu, X. Xue, H. Qin, C. Fan, Y. Wang, Q. Zhang, Bioorg. Med. Chem. Lett. 26, 849 (2016).

[5] P. S. Humphries, R. Bersot, J. Kincaid, E. Mabery, K. McCluskie, T. Park, T. Renner, E. Riegler, T. Steinfeld, E. D. Turtle, Z. L. Wei, E. Willis, Bioorg. Med. Chem. Lett. 26, 757 (2016).

[6] A. Mohry, F. Vogtle, M. Nieger, H. Hupfer, Chirality. 12, 76 (2000).

[7] Y. Luo, K. M.Qiu, X. Lu, K. Liu, J. Fu, H. L. Zhu. Bioorg. Med. Chem. 19, 4730 (2011)

[8] N. S. Reddy, M. R. Mallireddigari, S. Cosenza, K. Gumireddy, S. C. Bell, E. P. Reddy, M. V. R. Reddy, Bioorg. Med. Chem. Lett. 14, 4093 (2004).

[9] O. S. Moustafa, R. A. Ahmad. Phosphorus. Sulfur. Silicon. Relat. Elem. 178, 475 (2003).

[10] D. Guianvarc'h, M. Duca, C. Boukarim, L. K. Berthier, S. Leonce, A Pierre, B. Pfeiffer, P. Renard, P. B. Arimondo, C. Monneret, D. Dauzonne, J. Med. Chem. 47, 2365 (2004). 
[11] V. O. Rogatchov, H. Bernsmann, P. Schwab, R. Frohlich,B. Wibbeling, P. Metz. Tetrahedron Lett. 43, 4753 (2002)

[12] M. K. Parai, G. Panda, K. Srivastava, S. K. Puri, Bioorg. Med. Chem. Lett. 18, $776(2008)$.

[13] S. J. Kim, M. H. Jung, K. H. Yoo, J. H. Cho, C. H. Oh. Bioorg. Med. Chem. Lett. 18, 5815 (2008).

[14] M. M. Ghorab, F. A. Ragab, M. M. Hamed, Arzneimittelforschung. 60, 141 (2010).

[15] A. M. Ahad, S. Zuohe, L. D. Cuny, S. A. Moses, L. L. Zhou, S. Zhang, G. Powis, E. J. Meuillet, E. A. Mash. Bioorg. Med. Chem. 19, 2046 (2011).

[16] E. S. Darwish, A. M. Abdel Fattah, F. A. Attaby, O. N. Al-Shayea. Int. J. Mol. Sci. 15, 1237 (2014).

[17] O. A. Fathalla, W. A. Zaghary, H. H. Radwan, S. M. Awad, M. S. Mohamed, Arch Pharm Res. 25, 258 (2002).

[18] N. Boechat, L. C. S. Pinheiro, O. A. S. Filho, I. C. Silva, Molecules. 16, 8083 (2011).

[19] J. Drews, Science. 287, 1960 (2000)

[20] C. T. Supuran, I Drugs: Invest. Drug. J. 5, 1075 (2002).

[21] C.T. Supuran, A. Scozzafava, A. Casini. Med. Res. Rev. 23, 146 (2003)

[22] C. T. Supuran, A. Scozzafava, Expert. Opin. Ther. Patents.12, 217 (2002)

[23] C. T. Supuran, A. Scozzafava, Curr. Med. Chem. Imm. Endoc. Metab. Agents. 1, 61 (2001)

[24] B. Loh, L. Vozzolo, B.J. Mok, C.C. Lee, R.J. Fitzmaurice, S. Caddick, A. Fassati, Chem. Biol. Drug. Des.75, 461 (2010)..

[25] V. K. Agrawal, R. Srivastava, P. V. Khadikar, Bioorg. Med. Chem. 9, 3287 (2001).

[26] P. Selvam, M. Chandramohan, E. De Clercq, M. Witvrouw, C. Pannecouque, Eur. J. Pharm. Sci. 14, 313 (2001).

[27] X. Pan, J. Gao, J. Liu, J. Lai, H. Jiang, G. Yuan, Green Chem. 17, 2015 (2015)
[28] R. Sridhar, B. Srinivas, V. P. Kumar, M. Narender, K. R. Rao, Adv. Synth. Catal. 349, 1873 (2007)

[29] M. Jafarpour, A. Rezaeifard, T. Golshani, Phosphorus. Sulfur. Silicon. Relat. Elem. 186, 140 (2011).

[30] A. P. Álvarez, T. Topala, F. Estevan, F. Sanz, G. A. Piña, Eur. J. Inorg. Chem. 82 (2016)

[31] M. Harmata, P. Zheng, C. Huang, M. G. Gomes, W. Ying, K. O. Ranyanil, G. Balan, N. L. Calkins, J. Org. Chem. 72, 683 (2007).

32] A. Kamal, D. Dastagiri, M. J. Ramaiah, J. S. Reddy, E. V. Bharathi, M. K. Reddy, M. V. P. Sagar, T. L. Reddy, S. N. C. V. L. Pushpavalli, M. P. Bhadra, Eur. J. Med. Chem. 46, 5817 (2011).

33] F. Tamaddon, A. Nasiri, S. Farokhi, Catal. Commun. 1477, 12 (2011).

[34] F. Tamaddon, M. R. Sabeti, A. A. Jafari, F. Tirgir, E. Keshavarz, J. Mol Catal. A: Chem. 351, 41 (2011).

[35] H. H. Kang, H. S. Rho, D. H. Kim, S. G. Oh, Tetrahedron. Lett. 44, 7225 (2003).

[36] S. Lakrout, H. Ktir, A. Amira, M. Berredjemand N. E. Aouf, RSC. Adv. 4, 16027 (2014)

[37] S. Dineshkumar, G. Thirunarayanan, P. Mayavel, I. Muthuvel, Ovidius Univ. Annals Chem. 27, 22 (2016.

[38] S. John Joseph, K. Ranganathan, R. Suresh, R. Arulkumaran, R. Sundararajan, D. Kamalakkannan, S. P. Sakthinathan, G. Vanangamudi, S. Dineshkumar, K. Thirumurthy, I. Muthuvel, G. Thirunarayanan, K. Viveksarathi, Mat. Sci and Appl. Chem. 34, 12 (2017).

[39] A.W. Bauer, W.M.M. Kirby, J.C. Sherris, M. Truck, Am. J.Clin Pathol.45, 493 (1966).

[40] G. Thirunarayanan, Arabian J. Chem. 10, S636 (2017).

[41] SP. Sakthinathan, R. Suresh, D. Kamalakkannan, V. Mala, K. Sathiyamoorthi, G. Vanangamudi, G. Thirunarayanan, J. Chil. Chem. Soc., 63(2), 3918 (2018). 